May 2006

\title{
Hypertension and kidney disease in Asia
}

Tazeen H. Jafar

Aga Khan University, tazeen.jafar@aku.edu

Follow this and additional works at: https://ecommons.aku.edu/pakistan_fhs_mc_med_nephrol Part of the Nephrology Commons

\section{Recommended Citation}

Jafar, T. H. (2006). Hypertension and kidney disease in Asia. Current Opinion in Nephrology and Hypertension, 15(3), 291-295.

Available at: https://ecommons.aku.edu/pakistan_fhs_mc_med_nephrol/27 


\title{
Hypertension and kidney disease in Asia
}

\author{
Tazeen H. Jafar ${ }^{\mathrm{a}, \mathrm{b}}$
}

\begin{abstract}
Purpose of review
Communicable diseases were traditionally the major cause of public health concern in Asian countries, most of which were less developed. With industrialization and associated lifestyle changes during the past few decades, however, noncommunicable diseases similar to those that affect Western societies have emerged in Asian countries. The purpose of the review was to examine recent evidence about the burden and factors associated with hypertension and chronic kidney disease (CKD) in Asian countries.

\section{Recent findings}

Hypertension has become one of the leading causes of mortality in Asia. Although its prevalence continues to rise, it remains under-diagnosed and under-treated. CKD is becoming increasingly common mainly due to an increase in risk factors such as high blood pressure, diabetes, and obesity. Treatment of advanced CKD is overwhelmingly burdensome in a resource poor environment. Barriers to early detection of CKD in Asians include the fact that equations to estimate the glomerular filtration rate have not been validated in this population, and the uncertainty about appropriate glomerular filtration rate cutoff values to define CKD.

\section{Summary}

Concerted efforts are needed to develop and implement cost-effective strategies for prevention and treatment of hypertension and CKD in Asian countries. More research is needed on these conditions in these populations.
\end{abstract}

\section{Keywords}

Asian, chronic kidney disease, hypertension

Curr Opin Nephrol Hypertens 15:291-295. (C) 2006 Lippincott Williams \& Wilkins.

${ }^{\mathrm{a}}$ Section of Nephrology, Department of Medicine and ${ }^{\mathrm{b}}$ Clinical Epidemiology Unit, Department of Community Health Sciences, Aga Khan University, Karachi, Pakistan

Correspondence to Tazeen H. Jafar, MD, MPH, Head, Section of Nephrology, Director, Clinical Epidemiology Unit, Associate Professor, Medicine and Community Health Sciences, Aga Khan University, PO Box 3500, Stadium Road, Karachi, 74800, Pakistan

Tel: +92 21 4930051, ext. 4818; fax: +92 21 4934294;

e-mail: tazeen.jafar@aku.edu

Current Opinion in Nephrology and Hypertension 2006, 15:291-295

Abbreviations

BMI body mass index

Cl confidence interval

CKD chronic kidney disease

CVD cardiovascular disease

GFR glomerular filtration rate

MDRD Modification of Diet in Renal Disease

(C) 2006 Lippincott Williams \& Wilkins 1062-4821

\section{Introduction}

Communicable diseases were in the past the main causes of death worldwide and life expectancy was often limited by uncontrolled epidemics. Improvement of sanitary conditions and living conditions as well as medical advances in terms of vaccination and antibiotics, however, have successfully reduced infant mortality and deaths from infectious diseases. On the other hand, adopting the lifestyle of industrialized nations - such as decreased physical activity and increased intake of dietary fats - have resulted in the emergence of noncommunicable diseases as major problems in developing countries. Cardiovascular and kidney diseases, cancer, diabetes, chronic pulmonary and mental diseases pose an immense burden for health systems in both developed as well as developing countries, many of which are in Asia [1]. In 1990, for example, the leading causes of disease burden were pneumonia, diarrheal diseases, and perinatal conditions. It is predicted that by 2020 noncommunicable disease will account for $80 \%$ of the global burden of disease, causing seven out of every 10 deaths in developing countries [2,3].

\section{Hypertension}

Hypertension is one of the most important modifiable risk factors for cardiovascular disease (CVD) and chronic kidney disease (CKD) [4,5]. The prevalence of hypertension in countries in the Asian region is rising [6], but rates of awareness and adequate control of blood pressure remain appallingly low [7]. National-level efforts to stem the rising tide of hypertension are lacking in most Asian countries.

Despite the publications of several hypertension treatment guidelines, primary care practitioners continue to grossly under-diagnose and under-treat hypertension [8]. It appears that Asians are especially susceptible to insulin resistance, which may in part be due to past history of under-nutrition or low birth weight [9]. The presence of insulin resistance compounds the deleterious impact of high blood pressure in a synergistic manner in this vulnerable population, which is now facing an explosion of diabetes in epidemic proportions [2,10]. Ominously, other risk factors predisposing to GVD and CKD such as tobacco use, dietary fat intake, and sedentary lifestyles are also becoming widespread [11].

During the past two decades, China, the most populous nation in the world, has witnessed a doubling of the number of deaths attributed to chronic diseases, in 
particular hypertension. In the recently conducted nationally representative China National Hypertension Survey Epidemiology Follow-up Study [12•] on 169871 adult men and women, the single most important factor identified in terms of magnitude of adjusted relative risk as well as attributable risk of death was hypertension.

The prevalence of hypertension continues to rise in China. In 1998, 24\% of population aged 35-59 years suffered from this condition - a $2.3 \%$ increase compared with 1992-1994. Among hypertensive Chinese subjects, $42.6 \%$ were aware of their hypertension, $31.1 \%$ were treated, and merely $6 \%$ were controlled [6].

Data from Indo-Asian countries suggest an even higher burden of hypertension. Results from the National Health Survey of Pakistan 1990-1994 indicate that about onethird of the general population in Pakistan aged 40 years or over suffered from high blood pressure. Moreover, $70 \%$ of hypertensive patients were unaware of their condition, and blood pressure control rates (3\%) were dismal. In fact, the vast majority of adults have never had their blood pressure checked $[7,13]$. This is a reflection of the existing healthcare systems in developing countries that are sensitized mainly towards providing acute, symptomatic treatment for infectious diseases, and are not accustomed to providing preventive care for chronic diseases.

Knowledge regarding cardiovascular health issues, including hypertension, is severely lacking in some Asian countries. These gaps in knowledge coupled with high illiteracy rates translate into inadequate preventive healthcare practices [14]. Lack of hypertension screening and control programs in these regions means that mortality from this condition is only expected to escalate further $[6,7]$.

\section{Risk factors for hypertension}

Most of the rise in the prevalence of hypertension in countries such as China, Thailand, India, Pakistan, and other Asian countries can be explained by changes in distributions of body-mass index (BMI). Saturated fat intake, insulin resistance, and blood cholesterol levels have also increased concomitantly $[15,16]$.

This issue may be more complex as the downstream adverse consequences at any given severity of risk factor may be worse in Asian populations. For example, compared with white children in the US, children in Pakistan had higher blood pressure at any given BMI level (Fig. 1) [17]. Evidence suggests that associations between BMI, percentage of body fat, and health risks may differ in Asian and Western populations [18]. It has been shown that in order to identify subjects at high risk of CVD, lower values for BMI and waist circumference should be used for Asian patients than the conventional cutoff values for Western subjects [19]. One proposed explanation for these observed differences in magnitude of risk among Asians is the effect of poverty and resultant malnutrition during intrauterine and early childhood years. There may, however, also be other unexplained factors at work [20].

\section{Chronic kidney disease: low glomerular filtration rate}

In parallel with the sky-rocketing prevalence of uncontrolled hypertension and diabetes in Asia, the burden of CKD as defined by a decreased level of estimated glomerular filtration rate (GFR) or albuminuria is also expected to rapidly escalate in this region.

Adverse outcomes of CKD include loss of kidney function, sometimes leading to kidney failure, and CVD.

Figure 1 Body mass index (BMI) specific blood pressure levels in children in Pakistan versus white children in the US

Data are on 5374 South Asian children aged $5-14$ years included in the National Health Survey of Pakistan (NHSP) (1990-1994) and 3389 white children in the US included in NHANES III (19881994). BMl cutoff values are based on the 25th, 50th, 75th and 95th percentiles of values in the NHSP data. SBP, systolic blood pressure; DBP, diastolic blood pressure. Reproduced with permission from Jafar et al. [17].

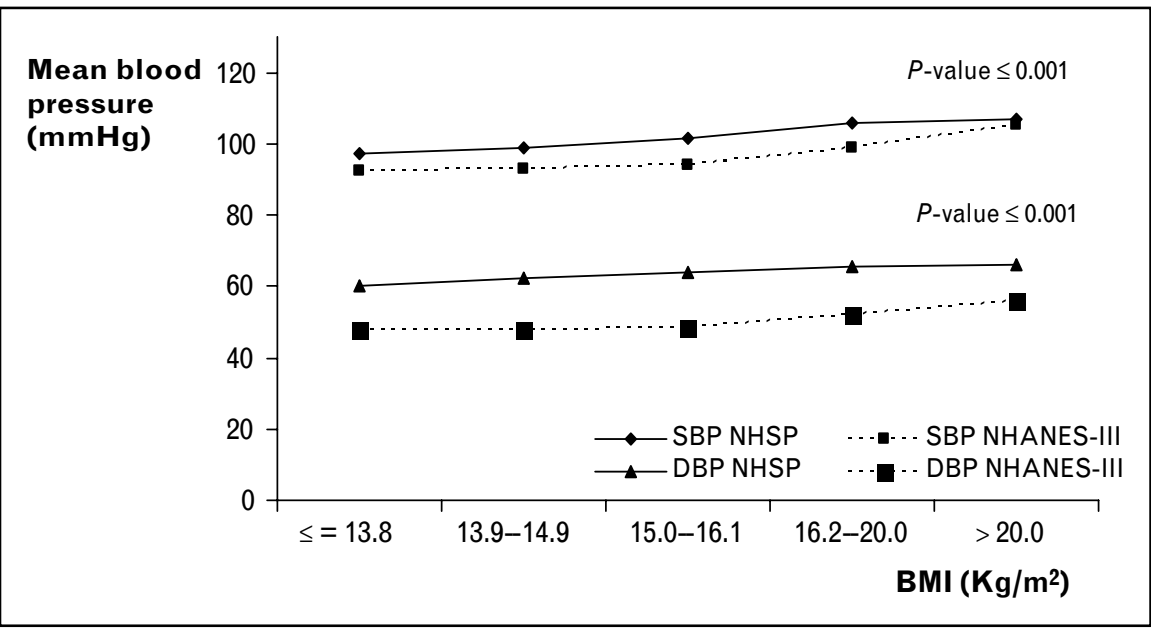


Unfortunately, CKD is under-diagnosed and under-treated [21,22]. This problem is compounded in developing countries that have poorly developed and underresourced healthcare system infrastructure. To give a concrete example, in Indo-Asian countries, the annual cost of dialysis is about US\$4000. Kidney transplantation cost about US $\$ 5000$, and post-transplant medications cost $\$ 2000$ annually. Compared with this, the average yearly per-capita income is $\$ 279 ; 36 \%$ of the population earns less than $\$ 105$ per annum, and only $2.2 \%$ earn more than $\$ 1000$ [23]. These countries therefore simply cannot afford to treat kidney failure. Mortality due to untreated CKD, although not well documented and quantified, is likely to be very high.

A recent cohort study [24] conducted over 12 years in Thailand on 3499 employees of the Electric Generation Authority suggested a steep rise in magnitude of CKD. In this study, the prevalence of decreased kidney function, defined as an estimated GFR less than $60 \mathrm{ml} / \mathrm{min}$ using the Modification of Diet in Renal Disease (MDRD) study equation, increased from $1.7 \%$ (95\% confidence interval [CI], 1.3-2.1) in 1985 to $6.8 \%$ (95\% CI, 5.7-7.9) in 1997. The prevalence of elevated serum creatinine $(>1.49 \mathrm{mg} / \mathrm{dl}$ in men and $>1.13 \mathrm{mg} / \mathrm{dl}$ in women) increased from $6.1 \%$ (95\% CI, 5.3-6.9) to $16.9 \%$ (95\% CI, 15.3-18.5). National level data were recently collected on serum creatinine levels during 2000 and 2001 on 15540 Chinese adults aged 35-74 years. The overall prevalence of reduced estimated GFR below $60 \mathrm{ml} / \mathrm{min}$ by the MDRD study equation was $2.53 \%$ [25].

It is important to point out in this context that the MDRD study and other serum-creatinine-based renal function estimating equations may not perform well among Asians. Data on 1166 adult subjects from the general population in Karachi, Pakistan collected during 2004 suggest that the prevalence of reduced estimated creatinine clearance $\left(<60 \mathrm{ml} / \mathrm{min}\right.$ per $\left.1.73 \mathrm{~m}^{2}\right)$ was
$21 \%$ in men and $30 \%$ in women aged 40 years or over if the Cockcroft-Gault equation were used. Using an MDRD equation estimated GFR cutoff value of $60 \mathrm{ml} /$ min per $1.73 \mathrm{~m}^{2}$, the prevalence of kidney disease was $9 \%$ in men and $11 \%$ in women, respectively (Table 1) [26]. A wide discrepancy in estimated disease prevalence is therefore obtained depending on whether the Cockcroft-Gault or the MDRD equation is used to estimate renal function in these participants. Agreement among GFR estimation equations and the measured GFR (or creatinine clearance) has been reported to be suboptimal in Asian populations $\left[27^{\bullet}, 28^{\circ}\right]$. A lower creatinine excretion in Indo-Asian men and women (17.5 and $14.1 \mathrm{mg} / \mathrm{kg}$ per day, respectively) has been reported compared with the expected values $(19.0$ and $16.3 \mathrm{mg} /$ kg per day; $P<0.002$ and $P<0.001$, respectively) in their Caucasian counterparts $\left[27^{\circ}\right]$. This may be explained by a lower body muscle mass and lower meat intake in Indo-Asians compared with white Caucasians. Improvements in the existing GFR estimation equations taking into account these differences are therefore needed for Asian populations.

Finally to complicate matters more, data from 610 young India potential kidney donors (with presumed normal kidney function) showed that the mean GFR values of Indians measuring using ${ }^{99} \mathrm{~m}$ Tc-diethylenetriamine pentaacetic acid plasma clearance was $81.4 \pm 19.4 \mathrm{ml} / \mathrm{min}$ per $1.73 \mathrm{~m}^{2}$ - significantly lower than what has been reported for their Western counterparts $(109-125 \mathrm{ml} / \mathrm{min}$ per $1.73 \mathrm{~m}^{2}$ body surface area) [29 $9^{\circ}$. This implies that even the GFR thresholds used to define CKD derived from Western subjects may need to be modified for Asian populations. Alternatively, kidney disease may be far more prevalent in this population than is currently appreciated.

More research is therefore needed to define population GFR norms, and to develop accurate renal function

Table 1 The age-specific prevalence (95\% confidence interval) of the reduced estimated glomerular filtration rate (GFR) in an IndoAsian population ${ }^{\mathrm{a}}$

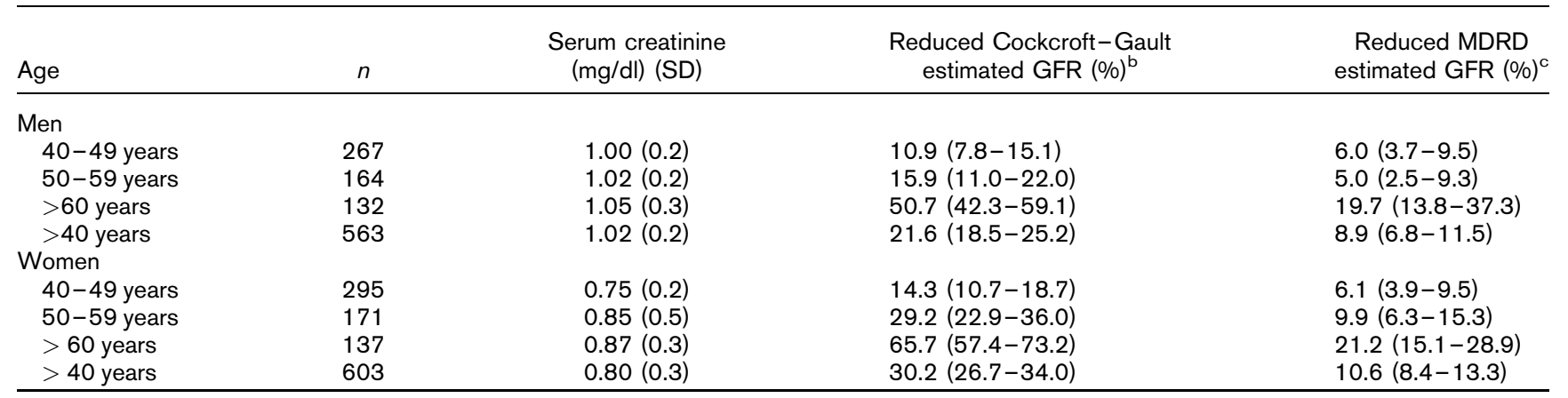

aThe reduced estimated GFR was defined as less than $60 \mathrm{ml} / \mathrm{min}$ per $1.73 \mathrm{~m}^{2}$.

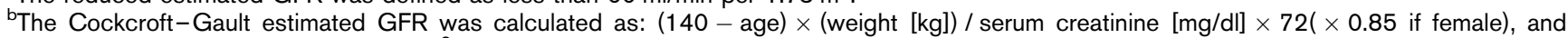
adjusted to body surface area of $1.73 \mathrm{~m}^{2}$.

${ }^{\mathrm{C}}$ The Modification of Diet in Renal Disease $\left(\right.$ MDRD) study estimated GFR was calculated as: $186 \times(\text { serum } \mathrm{creatinine}[\mathrm{mg} / \mathrm{dl}])^{-1.154} \times$ $(\text { age })^{-0.203}(\times 0.742$ if female $)$. 
estimation equations applicable to Asian populations from serum creatinine and other markers.

The general paucity of clinical trials conducted in indigenous Asian populations as well as lack of Asian subjects in those conducted in the West raise similar concerns about the applying these treatment targets and interventions strategies to Asian populations.

\section{Chronic kidney disease: albuminuria}

The other prominent aspect of CKD - albuminuria also seems to be highly prevalent across Asia. This is not unexpected given the increasing prevalence of diabetes in this region. In the Microalbuminuria Prevalence Study [30], the prevalence of macroalbuminuria was $18.8 \%$ and microalbuminuria was $39.8 \%$ among 6800 hypertensive diabetic adult patients from 10 Asian countries.

Although national-level general population data based on sound sampling strategies are needed for accurate assessment of burden of albuminuria and various stages of CKD in Asian countries, available evidence suggests a very high prevalence of CKD, and its risk factors in the Asian Pacific region.

\section{Risk factors for CKD}

Studies suggest that the main risk factors associated with CKD in the Asian populations include elevated blood pressure, elevated blood glucose and cholesterol levels, smoking, and high BMI $\left[24,31,32,33^{\bullet}, 34\right]$. Most of these are similar to those identified in Western populations $[35,36]$. The prevalence of CKD also appears to increase with increase in age in Asian countries [31]. To a significant extent this is due to the acquisition of the aforementioned risk factors, which are also associated with aging and often confound the association between age and CKD [24].

An as yet unresolved issue that relates to the Asian population is that of possible greater susceptibility of CKD because of a higher background prevalence of low birth weight or malnutrition [37-39]. A number of regional and global efforts have led to an overall improvement in the latter trends [40,41], and these efforts must continue. On the other hand, conventional risk factors for CKD are on the rise in Asia so programs that focus on prevention of CKD targeting conventional modifiable risk factors, including high blood pressure, diabetes, cholesterol, tobacco cessation, reduction in intake of saturated fats, and obesity, are urgently needed in Asian countries.

\section{Future direction for research in Asians}

More research is needed in the Asian population, mainly focusing on cost-effective strategies for early detection and prevention of hypertension, and CKD. This would include understanding the feasibility and efficacy of screening programs. Efforts are needed to study normal levels of GFR, and to develop and validate GFR estimation equations from serum creatinine and other markers that are applicable in this population. Prospective, well controlled studies are needed in the Asian populations to define appropriate target levels of risk factors, including the optimal level of BMI, waist circumference, cholesterol levels, blood pressure levels, and urine albumin excretion for prevention of morbidity and mortality from hypertension and CKD.

\section{Conclusion}

Hypertension is one the leading causes of mortality in Asia. Despite rising prevalence it remains under-diagnosed and under-treated. CKD is becoming increasingly common mainly due to an increase in conventional risk factors including high blood pressure, diabetes, and obesity. Early detection of CKD in Asians is challenging as equations to estimate GFR have not been validated in this population.

It is clear that well designed, cost-effective programs are needed to control hypertension and CKD, targeting their risk factors. These should include multiple strategies for primary and secondary prevention. Efforts need to be made to integrate these programs into the existing healthcare infrastructure for ensuring long-term sustainability.

Public education about adoption of healthy lifestyles must be one of the essential aims of such programs. It must, however, be coupled with appropriate public policy interventions that ensure affordability and access of such choices (e.g. antitobacco legislation and ban on sale of saturated oil), especially in countries where literacy rates are poor [11]. Cheap and reliable screening tests need to be used for population screening. Industry support is desired for subsidization of cost of effective medications, which are necessary for many high-risk individuals [42].

\section{References and recommended reading}

Papers of particular interest, published within the annual period of review, have been highlighted as:

- of special interest

- of outstanding interest

Additional references related to this topic can also be found in the Current World Literature section in this issue (p. 328).

1 Murray CJ, Lopez AD. Mortality by cause for eight regions of the world: global burden of disease study. Lancet 1997; 349:1269-1276.

2 Boutayeb A, Boutayeb S. The burden of non communicable diseases in developing countries. Int J Equity Health [online journal] 2005; 4:2. http:// www.equityhealthj.com/content/4/1/2.

3 Lenfant C. Can we prevent cardiovascular diseases in low- and middleincome countries? Bull World Health Org 2001; 79:980-982; discussion 983-987.

4 Hypertension Detection and Follow-up Program Cooperative Group. Fiveyear findings of the hypertension detection and follow-up program. I. Reduction in mortality of persons with high blood pressure, including mild hypertension. Hypertension Detection and Follow-up Program Cooperative Group. 1979. JAMA 1997; 277:157-166. 
5 Jafar TH, Stark PC, Schmid CH, et al. Progression of chronic kidney disease: the role of blood pressure control, proteinuria, and angiotensin-converting enzyme inhibition: a patient-level meta-analysis. Ann Intern Med 2003; 139:244-252.

6 Wang $Z, W u Y, Z$ hao $L$, et al. Trends in prevalence, awareness, treatment and control of hypertension in the middle-aged population of China, 1992-1998. Hypertens Res 2004; 27:703-709.

7 Ahmad K, Jafar TH. Prevalence and determinants of blood pressure screening in Pakistan. J Hypertens 2005; 23:1979-1984.

8 Jafar TH, Jessani S, Jafary FH, et al. General practitioners' approach to hypertension in urban Pakistan: disturbing trends in practice. Circulation 2005; 111:1278-1283.

9 Bhargava SK, Sachdev HS, Fall CH, et al. Relation of serial changes in childhood body-mass index to impaired glucose tolerance in young adulthood. N Engl J Med 2004; 350:865-875.

10 Gu D, Reynolds K, Duan X, et al. Prevalence of diabetes and impaired fasting glucose in the Chinese adult population: International Collaborative Study of Cardiovascular Disease in Asia (Interasia). Diabetologia 2003; 46:11901198.

11 Reddy KS. Cardiovascular disease in non-western countries. N Engl J Med 2004; 350:2438-2440

$12 \mathrm{He} \mathrm{J,} \mathrm{Gu} \mathrm{D,} \mathrm{Wu} \mathrm{X,} \mathrm{et} \mathrm{al.} \mathrm{Major} \mathrm{causes} \mathrm{of} \mathrm{death} \mathrm{among} \mathrm{men} \mathrm{and} \mathrm{women} \mathrm{in}$ •• China. N Engl J Med 2005; 353:1124-1134.

This is a well conducted 10-year follow-up study on 169871 subjects in China in which hypertension had the highest multivariate-adjusted relative risk as well as the population attributable risk for death.

13 Jafar TH, Levey AS, Jafary FH, et al. Ethnic subgroup differences in hypertension in Pakistan. J Hypertens 2003; 21:905-912.

14 Jafary $\mathrm{FH}$, Aslam $\mathrm{F}$, Mahmud $\mathrm{H}$, et al. Cardiovascular health knowledge and behavior in patient attendants at four tertiary care hospitals in Pakistan: a cause for concern. BMC Public Health [online journal] 2005; 5:124.

15 Silventoinen $\mathrm{K}$, Sans $\mathrm{S}$, Tolonen $\mathrm{H}$, et al. Trends in obesity and energy supply in the WHO MONICA Project. Int J Obes Relat Metab Disord 2004; 28:710718.

16 The World Health Report 2002. Reducing risks, promoting healthy life. Geneva: World Health Organization; 2002.

17 Jafar TH, Islam M, Poulter N, et al. Children in South Asia have higher body mass-adjusted blood pressure levels than white children in the United States: a comparative study. Circulation 2005; 111:1291-1297.

18 WHO Expert Consultation. Appropriate body-mass index for Asian populations and its implications for policy and intervention strategies. Lancet 2004; 363:157-163.

19 Wildman RP, Gu D, Reynolds K, et al. Appropriate body mass index and waist circumference cutoffs for categorization of overweight and central adiposity among Chinese adults. Am J Clin Nutr 2004; 80:1129-1136.

20 Fuller KE. Low birth-weight infants: the continuing ethnic disparity and the interaction of biology and environment. Ethnic Dis 2000; 10:432445.

21 Kausz AT, Obrador GT, Arora P, et al. Late initiation of dialysis among women and ethnic minorities in the United States. J Am Soc Nephrol 2000; 11:2351-2357

22 Mcclellan WM, Knight DF, Karp H, Brown WW. Early detection and treatment of renal disease in hospitalized diabetic and hypertensive patients: important differences between practice and published guidelines. Am J Kidney Dis 1997; 29:368-375

23 Mani MK. Prevention of chronic renal failure at the community level. Kidney Int 2003; Suppl:S86-S89.

24 Domrongkitchaiporn S, Sritara P, Kitiyakara C, et al. Risk factors for development of decreased kidney function in a southeast Asian population: a 12-year cohort study. J Am Soc Nephrol 2005; 16:791-799.
25 Chen J, Wildman RP, Gu D, et al. Prevalence of decreased kidney function in chinese adults aged 35 to 74 years. Kidney Int 2005; 68:2837-2845.

26 Jafar TH, Hatcher J, Chaturvedi N, Levey AS. Prevalence of reduced estimated GFR (eGFR) in Indo Asian population [abstract]. J Am Soc Nephrol 2005; 16:323A.

27 Jafar $\mathrm{TH}$, Schmid $\mathrm{CH}$, Levey AS. Serum creatinine as marker of kidney

- function in South Asians: a study of reduced GFR in adults in Pakistan. J Am Soc Nephrol 2005; 16:1413-1419.

A study in a Pakistani population showing suboptimal agreement between estimated and measured GFR ( $24 \mathrm{~h}$ creatinine clearance), and a lower mean creatinine excretion in these subjects, which may, in part, account for the above.

28 Zuo L, Ma YC, Zhou YH, et al. Application of GFR-estimating equations in

- Chinese patients with chronic kidney disease. Am J Kidney Dis 2005; 45:463-472.

A study assessing the validity of the MDRD study GFR estimation equation in a Chinese population. Poor agreement was found between the estimated and measured GFR, especially at high and low levels of the latter.

29 Barai S, Bandopadhayaya GP, Patel CD, et al. Do healthy potential kidney - donors in india have an average glomerular filtration rate of $81.4 \mathrm{ml} / \mathrm{min}$ ? Nephron Physiol 2005; 101:21-26.

Interesting study on healthy kidney donors from India using the ${ }^{99} \mathrm{mTc}$-diethylenetriamine pentaacetic acid two-plasma sample method of Russell. The mean GFR value of a young healthy Indian adult potential kidney donor is $81.4 \pm 19.4 \mathrm{ml} / \mathrm{min}$ per $1.73 \mathrm{~m}^{2}$ body surface area, which is significantly different from the normal value of $109-125 \mathrm{ml} / \mathrm{min}$ per $1.73 \mathrm{~m}^{2}$ body surface area derived from a Western population.

30 Weir MR. Albuminuria predicting outcome in diabetes: incidence of microalbuminuria in Asia-Pacific Rim. Kidney Int 2004; Suppl:S38-S39.

31 Jafar TH, Chaturvedi N, Gul A, et al. Ethnic differences and determinants of proteinuria among South Asian subgroups in Pakistan. Kidney Int 2003; 64:1437-1444

32 Yamada $\mathrm{Y}$, Noborisaka $\mathrm{Y}$, Ishizaki $\mathrm{M}$, et al. Association between cigarette consumption and proteinuria in healthy Japanese men and women from an occupational population. J Occup Health 2004; 46:365-373.

33 Jee SH, Boulware LE, Guallar E, et al. Direct, progressive association of

- cardiovascular risk factors with incident proteinuria: results from the Korea Medical Insurance Corporation (KMIC) study. Arch Intern Med 2005; $165: 2299-2304$

A prospective study over 10 years of incident proteinuria in Korean adults. Fasting glucose and cholesterol levels, BMI, and blood pressure were identified as independent predictors.

34 Iseki K. Predictors of diabetic end-stage renal disease in Japan. Nephrology (Carlton) 2005; 10 (Suppl):S2-S6.

35 Stengel B, Tarver-Carr ME, Powe NR, et al. Lifestyle factors, obesity and the risk of chronic kidney disease. Epidemiology 2003; 14:479-487.

36 Mcclellan WM. Epidemiology and risk factors for chronic kidney disease. Med Clin North Am 2005; 89:419-445.

37 Ramirez SP, Hsu SI, Mcclellan W. Low body weight is a risk factor for proteinuria in multiracial Southeast Asian pediatric population. Am J Kidney Dis 2001; 38:1045-1054.

38 Ramirez SP, Mcclellan W, Port FK, Hsu SI. Risk factors for proteinuria in a large, multiracial, Southeast Asian population. J Am Soc Nephrol 2002; 13:1907-1917.

39 Wani M, Kalra V, Agarwal SK. Low birth weight and its implication in renal disease. J Assoc Physicians India 2004; 52:649-652.

40 Antonisamy B, Sivaram M, Richard J, Rao PS. Trends in intra-uterine growth of single live births in Southern India. J Trop Pediatr 1996; 42:339-341.

41 Chang $Y$, Xian ZD, He W, et al. Child malnutrition in China: present status and changing trend. Biomed Environ Sci 1996; 9:164-180.

42 Wang YR. The Chinese pharmaceutical market at the crossroads: procompetition solutions to improve access, quality and affordability. Appl Health Econ Health Policy 2005; 4:147-151. 